\title{
Tecnologia educacional: A enfermagem e os jogos educativos na educação em saúde
}

\author{
Educational technology: Nursing and educational games in health education \\ Tecnología educativa: Enfermería y juegos educativos em educación para la salud
}

Recebido: 24/05/2021 | Revisado: 30/05/2021 | Aceito: 01/06/2021 | Publicado: 15/06/2021

Isabelle Christine Nunes de Carvalho

ORCID: https://orcid.org/0000-0002-9671-3907 Universidade Federal do Estado do Rio de Janeiro, Brasil E-mail: isa.cnc29@gmail.com

Monique Oliveira de Freitas Nascimento

ORCID: https://orcid.org/0000-0002-6711-098X Hospital Norte D'or, Brasil

E-mail: moniquefreitas2402@gmail.com

Ana Cristina Silva Pinto

ORCID: https://orcid.org/0000-0002-5608-2418 Universidade Federal do Estado do Rio de Janeiro, Brasil E-mail: ana.pinto@unirio.br

Elizabeth Rodrigues Fonseca de Melo

ORCID: https://orcid.org/0000-0002-0639-8326 Hospital Norte D'or, Brasil

E-mail: rodrigues.enf85@hotmail.com

Giselle Regine Nunes de Carvalho

ORCID: https://orcid.org/0000-0003-2949-6567

Hospital Municipal Souza Aguiar, Brasil E-mail: gihrnc@gmail.com

Maria Célia Teixeira dos Santos

ORCID: https://orcid.org/0000-0002-7490-5537

Universidade Federal do Estado do Rio de Janeiro, Brasil E-mail: celiat.barbosa@gmail.com

\begin{abstract}
Resumo
O objetivo deste estudo foi analisar as produções científicas voltadas para os jogos educativos que são utilizados para pacientes e familiares na educação em saúde. Os jogos educativos em saúde podem ser aplicados em diversas situações segundo a literatura, podendo ser utilizado como um recurso pedagógico e facilitando o processo de ensino dos profissionais de saúde. Este estudo é baseado na pesquisa bibliográfica tendo como base a revisão integrativa com o intuito de obter uma avaliação crítica da produção como estratégia para a síntese dos dados para o estudo. Analisando através desta amostra de 17 artigos, selecionados a partir dos critérios traçados, o número de publicações por ano em que foram publicadas temos: 2019 apresentando 07 publicações $(41,1 \%)$, seguindo do ano de 2018 com 06 estudos (35,2\%), 2020 com 03 (17,6\%) e 2017 com 01(5,8\%). Portanto pode-se concluir que o ano em que mais se publicou sobre a temática foi o ano de 2018 com $29,54 \%$ dos estudos. Com este estudo, concluiu-se que embora os jogos sejam ferramentas de grande utilidade na área da educação em saúde ainda há a necessidade de um maior aprofundamento nos estudos acerca do tema.
\end{abstract}

Palavras-chave: Tecnologia educacional; Enfermagem; Jogos e brincadeiras; Educação em saúde; Ensino.

\begin{abstract}
The objective of this study was to analyze the scientific productions focused on educational games that are used for patients and families in health education. According to the literature, health educational games can be applied in several situations, and can be used as a pedagogical resource, facilitating the teaching process of health professionals. This study is based on bibliographic research with integrative review as a basis, in order to obtain a critical evaluation of the production as a strategy to synthesize data for the study. Analyzing through this sample of 17 articles, selected from the drawn criteria, the number of publications per year in which they were published we have: 2019 presenting 07 publications (41.1\%), followed by the year 2018 with 06 studies (35.2\%), 2020 with 03 (17.6\%) and 2017 with 01 (5.8\%). Therefore, it can be concluded that the year in which the most published about the theme was the year 2018 with $29.54 \%$ of studies. With this study, it was concluded that although games are very useful tools in the area of health education, there is still a need for further study on the subject.
\end{abstract}

Keywords: Educational Technology; Nursing; Play and Playthings; Health Education; Teaching. 


\section{Resumen}

El objetivo de este estudio fue analizar las producciones científicas centradas en los juegos educativos que se utilizan para los pacientes y las familias en la educación sanitaria. Los juegos educativos de salud pueden aplicarse en diversas situaciones, según la literatura, y pueden utilizarse como recurso pedagógico, facilitando el proceso de enseñanza de los profesionales de la salud. Este estudio se basa en la investigación bibliográfica basada en la revisión integradora para obtener una evaluación crítica de la producción como estrategia de síntesis de datos para el estudio. Analizando a través de esta muestra de 17 artículos, seleccionados a partir de los criterios elaborados, el número de publicaciones por año en el que fueron publicados tenemos: 2019 presentando 07 publicaciones $(41,1 \%)$, seguido del año 2018 con 06 estudios (35,2\%), 2020 con $03(17,6 \%)$ y 2017 con $01(5,8 \%)$, por lo que se puede concluir que el año en el que más se publica sobre el tema fue el año 2018 con 29,54\% de estudios. Concluyó que si bien los juegos son herramientas muy útiles en el área de la educación para la salud, aún es necesario profundizar en el estudio sobre el tema.

Palabras clave: Tecnología educativa; Enfermería; Paly y juguetes; Educación para la salud; Ensenãnza.

\section{Introdução}

A educação em saúde é dada por um processo no qual profissionais que atuam neste setor buscam formas de estimular os indivíduos a desenvolver o senso de responsabilidade relacionado aos cuidados com a própria saúde. Esse estímulo pode ser dado através da realização de atividades lúdicas como jogos que são instrumentos educativos que apresentam uma grande capacidade em auxiliar na construção do conhecimento em saúde de modo que vise o esclarecimento de dúvidas facilitando o processo de aprendizagem do ser humano de forma inovadora, estimulante e divertida (Costa, Ermini \& Sigaud, 2019).

A utilização de jogos em educação à saúde busca proporcionar o aprendizado de modo mais lúcido permitir a troca de experiências, além de facilitar a socialização e reflexão da cultura e da importância de cuidados à saúde e prevenção de doenças. Os jogos tendem a permitir uma melhor discussão em determinados grupos sobre temas relevantes à ele (Fonseca, Santo, Silva \& Silvino, 2015).

O objetivo deste estudo foi analisar as produções científicas em enfermagem voltadas para os jogos educativos que são utilizados para pacientes e familiares na educação em saúde.

Os jogos educativos em saúde podem ser aplicados em diversas situações segundo a literatura, podendo ser utilizados como um recurso pedagógico e facilitando o processo de ensino dos profissionais de saúde (Brandão, et al., 2019).

Dessa forma, é possível afirmar que os jogos educativos estimulam o aprendizado dos indivíduos, valorizando suas experiências e incentivando o resgaste do diálogo entre profissionais e os seus participantes utilizando-se da construção do conhecimento através de debates e outras formas de expressão para suas ideias (Rosi, et al., 2016).

\section{Metodologia}

Foi realizada uma busca na literatura, através de revisão integrativa, que para Tossin et al. (2016) tem por intuito de avaliação crítica da produção e como estratégia para síntese dos dados. Podendo assim ter um panorama do que há publicado sobre o assunto a ser tratado. Pesquisa de caráter qualitativo por interpretar o fenômeno em estudo sob a ótica do pesquisador (Pereira, Shitsuka, Parreira \& Shitsuka, 2018).

Seguiu-se as etapas descritas por Mendes, Silveira \& Galvão (2008): identificação do tema e seleção da questão de pesquisa, delimitação dos critérios para inclusão e exclusão de estudos, identificação dos estudos pré-selecionados e selecionados, categorização dos estudos, análise e interpretação dos resultados e apresentação da revisão/síntese do conhecimento.

Desta forma, a pergunta norteadora do estudo foi: "Qual é a produção científica de enfermagem sobre jogos educativos utilizados para educação em saúde de pacientes e familiares?". Para construir esta pergunta houve a utilização do acrônimo PICo, que segundo Sousa, Marques, Firmino, Frade, Valentim e Antunes (2018): P de população (Pacientes e familiares); I de interesse/ fenômeno (jogos educativos); Co de contexto (educação em saúde). 
Para obter o resultado foram cruzados par a par os descritores (DECS/MESH): "Educational Technology", "Nursing", "Play and Playthings" e "Health education". Justificando-se pelo objetivo de alcançar estudos que abordagem a utilização de jogos como tecnologias educacionais para promoção da educação em saúde. Utilizou-se o operador booleano “AND” para cada uma das bases de dados selecionadas e como eixo norteador a pergunta e os critérios de inclusão estabelecidos. A busca foi realizada através do acesso online.

Ano: As limitações quanto ao ano de publicação dos artigos durante as buscas realizadas foi de 2017 a abril de 2021 , obtendo assim o que há de mais atual disponível na literatura.

Periódicos: Foram analisadas quatro bases de dados: CINAHL, SCOPUS, WEB OF SCIENCE e MEDLINE, para que houvesse um apanhado no contexto internacional.

Critérios de inclusão: Os critérios de inclusão foram artigos de pesquisa original, completo e publicado nos idiomas Português, Inglês ou Espanhol; que abordassem o uso de jogos utilizados com intuito de orientação relacionada à promoção da educação em saúde.

Critérios de exclusão: Foram excluídos desta revisão estudos que não abordassem atuação do enfermeiro, aqueles que não estivessem disponibilizados na íntegra, teses e dissertações, assim como aos que não atendiam ao objetivo deste estudo.

\section{Resultados e Discussão}

A busca dos artigos através do cruzamento dos descritores, pode ser explicitada a seguir:

Fluxograma 1: Percurso metodológico da busca dos artigos.

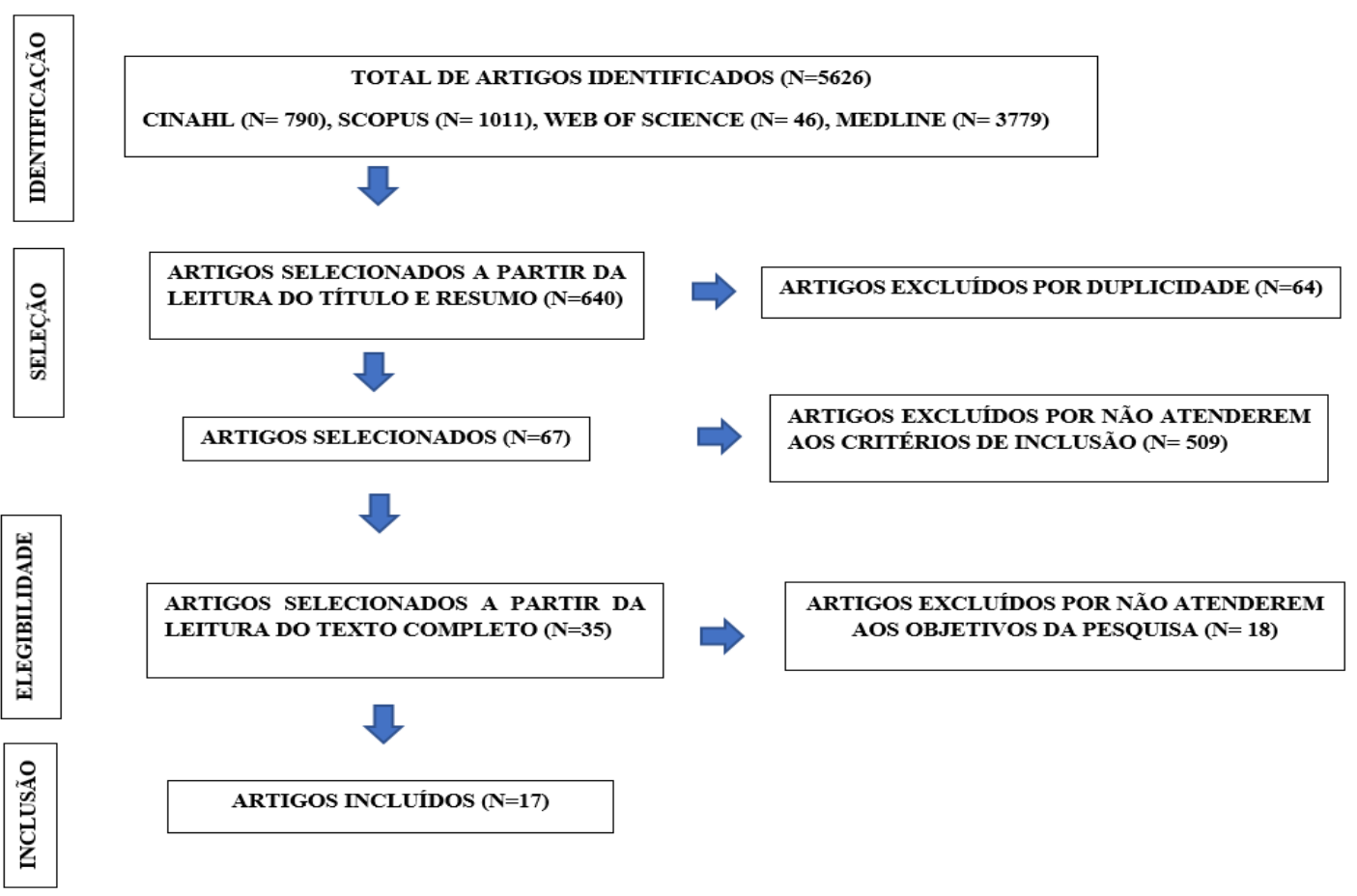

Fonte: Autores (2021).

O compilamento dos dados ocorreu mediante tabela objetivando evidenciar as características das pesquisas. Esta tabela segue em anexo ao artigo.

Através da busca pelo cruzamento dos descritores par a par obteve-se os seguintes resultados. 
Quadro 1: Publicações provenientes da estratégia de busca utilizada.

\begin{tabular}{|c|c|c|c|c|}
\hline BASE DE DADO & ANO & TíTULO & CLIENTELA & CONTRIBUIÇÕES \\
\hline CINAHL & 2019 & $\begin{array}{l}\text { Addressing mal nutrition and access to food for } \\
\text { older people: the Food In Later Life Game }\end{array}$ & Idosos & $\begin{array}{l}\text { Pode tornar o aprendizado memorável, agradável e eficaz. Incentivar a } \\
\text { discussão de novas ideias, compartilhamento de conhecimento e aprendizado } \\
\text { uns com os outros. }\end{array}$ \\
\hline CINAHL & 2019 & \begin{tabular}{|c|} 
Parental Knowledge Influenced the Effectiveness \\
of Therapeutic Sociodramatic Play on Diarrheal \\
Preventive Behavior in School-Age Children
\end{tabular} & $\begin{array}{l}\text { Crianças } \\
\text { escolares }\end{array}$ & $\begin{array}{l}\text { Reflete acontecimentos reais, podendo motivar as crianças a agir de acordo } \\
\text { com o que elas experimentaram durante de papéis jogo. } \\
\text { O jogo afeta os conhecimentos, atitudes e habilidades de crianças em idade } \\
\text { escolar. }\end{array}$ \\
\hline CINAHL & 2019 & $\begin{array}{l}\text { A Demonstration Project for the Utility of Kinect- } \\
\text { Based Educational Games to Benefit Motor Skills } \\
\text { of Children with ASD. }\end{array}$ & $\begin{array}{l}\text { Crianças com } \\
\text { TEA }\end{array}$ & $\begin{array}{c}\text { Os jogos educacionais são projetados para incentivar a assimilação de novos } \\
\text { conceitos e deve tentar reduzir a carga de esforço enquanto aumenta a } \\
\text { motivação e os benefícios da aprendizagem. }\end{array}$ \\
\hline SCOPUS & 2019 & $\begin{array}{l}\text { Play and Learn with Patients_-Designing and } \\
\text { Evaluating a Serious Game to Enhance Nurses' } \\
\text { Inhaler Teaching Techniques: a randomized } \\
\text { controlled trial. }\end{array}$ & $\begin{array}{l}\text { Enfermeiros e } \\
\text { pacientes com } \\
\quad \text { asma }\end{array}$ & $\begin{array}{l}\text { fornecer educação. } \\
\begin{array}{l}\text { O estudo demonstrou a eficácia do jogo sério em melhorar a autoeficácia e o } \\
\text { desempenho no ensino da técnica inalatória. }\end{array} \\
\text { Solução prática de aprendizagem, devido favorecimento do aprendizado }\end{array}$ \\
\hline SCOPUS & 2018 & $\begin{array}{l}\text { Giving Voice to Caregivers: A game for family } \\
\text { caregivers of dependent individuals }\end{array}$ & $\begin{array}{l}\text { Cuidador } \\
\text { Familiar }\end{array}$ & $\begin{array}{c}\text { estimular mudanças comportamentais. } \\
\text { Permite criação de um local para reflexão, partilha de valores e ações que } \\
\text { orientam sentimentos e emoções, minimizando diferenças e permitindo } \\
\text { mediar a negociação de acordos. }\end{array}$ \\
\hline SCOPUS & 2020 & $\begin{array}{l}\text { MANTRA: development and localization of a } \\
\text { mobile educational health game targeting low } \\
\text { literacy players in low and middle income } \\
\text { countries. }\end{array}$ & $\begin{array}{l}\text { Mulheres com } \\
\text { baixo nível de } \\
\text { alfabetização }\end{array}$ & \begin{tabular}{|} 
O jogo tem a visão transmitir conhecimento. Através da discussão entre a \\
comunidade, partindo de uma demanda dos jogadores. \\
Fortaleceu a confiança na abordagem das comunidades. \\
A motivação e a facilidade para aprender através do jogo foram pontuados pelos \\
participantes. \\
$\begin{array}{c}\text { Destacou-se também o potencial do jogo de expansão para outras funções com } \\
\text { intuito de apoiar mudança de comportamentos de risco }\end{array}$
\end{tabular} \\
\hline NEB OF SCIENCE & 2019 & $\begin{array}{l}\text { Electronic games in child and adolescent health } \\
\text { care: an integrative review }\end{array}$ & $\begin{array}{c}\text { Crianças e } \\
\text { adolescentes }\end{array}$ & $\begin{array}{c}\text { O estudo demonstrou relevância com uso da tecnologia de jogos eletrônicos, } \\
\text { onde por meio da educação em saúde possibilitou a caminhada para } \\
\text { melhoraria/controle de morbidades e complicações. } \\
\text { Permitiram aprendizado favorecendo mudança de hábitos, melhora cognitiva, } \\
\text { desenvolvimento de habilidades, autoconhecimento, facilitou a compreensão } \\
\text { de quadros clínicos de doenças crônicas. }\end{array}$ \\
\hline MEDLINE & 2019 & $\begin{array}{l}\text { Kaizen: Interactive Gaming for Diabetes Patient } \\
\text { Education. }\end{array}$ & $\begin{array}{l}\text { Pacientes } \\
\text { diabéticos }\end{array}$ & $\begin{array}{c}\text { O aprendizado baseado em jogos permite maior envolvimento em cada consulta } \\
\text { e discussões mais significativas conduzidas pelo paciente, ou seja, participação } \\
\text { mais efetiva nas consultas e consequentemente em seu cuidado. }\end{array}$ \\
\hline MEDLINE & 2018 & $\begin{array}{l}\text { Board games: gerotechnology in nursing care } \\
\text { practice. : gerotechnology in nursing care } \\
\text { practice. }\end{array}$ & Idosos & $\begin{array}{c}\text { O jogo mostrou-se como uma tecnologia que facilita a interação entre os } \\
\text { educandos e educadores, proporcionando o processo de ensino-aprendizagem, } \\
\text { auxiliando na tomada de decisões. Sinalizam a importância no investimento do } \\
\text { uso de tecnologias para manutenção da autonomia da pessoa visando o } \\
\text { autocuidado. }\end{array}$ \\
\hline MEDLINE & 2020 & $\begin{array}{l}\text { Testing the Usability of Digital Educational } \\
\text { Games for Encouraging Smoking Cessation. }\end{array}$ & Tabagistas & $\begin{array}{l}\text { Os jogos educacionais digitais foram pontuados como fáceis de utilizar, úteis e } \\
\text { satisfatórios, além de a oferta de conteúdo por este meio ser benéfica para } \\
\text { foco e concentração, aumentou-se a motivação para mudança no } \\
\text { comportamento. }\end{array}$ \\
\hline MEDLINE & 2018 & $\begin{array}{c}\text { Serious games as an educational strategy to } \\
\text { control childhood obesity: a systematic literature } \\
\text { review. }\end{array}$ & Crianças & $\begin{array}{c}\text { Os jogos sérios utilizados como estratégia para mudanças de comportamentos } \\
\text { estão em expansão e sua utilização revelou resultados promissores. Além de } \\
\text { sua eficácia na melhoria do conhecimento, no incentivo a mudanças de } \\
\text { comportamentos ou até melhoria resultados à saúde. }\end{array}$ \\
\hline MEDLINE & 2017 & $\begin{array}{l}\text { The game as strategy for approach to sexuality } \\
\text { with adolescents: theoretical-methodological } \\
\text { reflections. }\end{array}$ & Adolescentes & $\begin{array}{c}\text { Traz o jogo como um dispositivo, que durante sua utilização permitiu } \\
\text { empoderamento e produção de singularidades. Potencializando o aprendizado e } \\
\text { atraindo os jogadores . Além disso, permite interação, favorecendo: construção } \\
\text { mais ativa, o processo de formação de conceitos de outros jogadores. }\end{array}$ \\
\hline MEDLINE & 2018 & $\begin{array}{c}\text { Digital Gaming for Improving the Functioning of } \\
\text { People With Traumatic Brain Injury: Randomized } \\
\text { Clinical Feasibility Study }\end{array}$ & $\begin{array}{l}\text { Pessoas com } \\
\text { lesão cerebral } \\
\text { traumática. }\end{array}$ & $\begin{array}{c}\text { Para perceber resultados válidos da implementação dos jogos precisa de doses } \\
\text { mais altas, ou seja, exposição maior, com monitoramento. }\end{array}$ \\
\hline MEDLINE & 2018 & $\begin{array}{l}\text { Effect of the board game as educational } \\
\text { technology on schoolchildren's knowledge on } \\
\text { breastfeeding } 1\end{array}$ & $\begin{array}{c}\text { Crianças e } \\
\text { adolescentes }\end{array}$ & $\begin{array}{l}\text { Indica que o jogo pode ser uma ferramenta eficaz no ensino de conteúdos. A } \\
\text { acessibilidade para levar o jogo para casa permitiu sessões adicionais com } \\
\text { familiares e amigos indicando possibilidade de maior agregação de } \\
\text { conhecimento. }\end{array}$ \\
\hline MEDLINE & 2019 & $\begin{array}{l}\text { Role of Digital Games in Self-Management of } \\
\text { Cardiovascular Diseases: A Scoping Review. }\end{array}$ & $\begin{array}{c}\text { Portadores } \\
\text { doença } \\
\text { cardiovascular. }\end{array}$ & \begin{tabular}{|c|} 
Os jogos despertaram como uma possibilidade à educação tradicional do \\
paciente e através da oferta de um caminho novo e excitante para aprender e se \\
envolver em comportamentos, além disso podem disponibilizar ambientes \\
educacionais flexíveis, acessíveis e atraentes.
\end{tabular} \\
\hline MEDLINE & 2020 & $\begin{array}{l}\text { Serious Game e-Baby Família: an educational } \\
\text { technology for premature infant care. }\end{array}$ & $\begin{array}{l}\text { Pais de crianças } \\
\text { prematuras }\end{array}$ & $\begin{array}{c}\text { Destaca o jogo sério como uma abordagem inovadora na educação para a saúde. } \\
\text { Os jogos de computador se destacam devido a possibilitarem desafio além de } \\
\text { envolver o participante. } \\
\text { Ratifica-se as vantagens da utilização do jogo, como: realismo, satisfação em } \\
\text { brincar e percepção de aprendizagem. }\end{array}$ \\
\hline MEDLINE & 2018 & $\begin{array}{c}\text { DECIDIX: meeting of the Paulo Freire pedagogy } \\
\text { with the serious games in the field of health } \\
\text { education with adolescents. }\end{array}$ & Adolescentes & $\begin{array}{l}\text { Destaca-se a construção de relações através do diálogo; com potencialização } \\
\text { do vínculo entre envolvidos, assim como a construção de experiências de } \\
\text { aprendizagem que possibilitem a autonomia dos atores envolvidos no contexto } \\
\text { da saúde sexual e reprodutiva. }\end{array}$ \\
\hline
\end{tabular}

Fonte: Autores (2021).

Analisando através desta amostra de 17 artigos, selecionados a partir dos critérios já citados, o número de publicações por ano em que foram publicadas temos: 2019 apresentando 07 publicações (41,1\%), seguindo do ano de 2018 com 06 estudos (35,2\%), 2020 com 03 (17,6\%) e 2017 com 01(5,8\%). Portanto, pode-se concluir que o ano em que mais se publicou sobre a temática foi o ano de 2018 com $29,54 \%$ dos estudos. 
Quanto à clientela que era o foco da utilização dos jogos, a maioria foi de crianças (apareceram em 05 artigos sendo a população alvo, 29,4\%) seguidos idosos e adolescentes com 02 artigos cada (11,7\% cada); família/cuidador aparecem em 02 artigos cada $(4,54 \%)$.

Com isso, pode-se perceber que a população que mais foi alvo da utilização de jogo para educação em saúde na literatura foram crianças. Exacerbando a necessidade de ocorreram mais estudos com a temática.

Cabe ressaltar também que na amostra só um artigo pontuava a utilização do jogo na beira do leito, aquele que a população asmática necessitava de orientações, trazendo mais este aspecto a ser considerado. A aplicação de jogos como TEs para educação em saúde no âmbito terciário, a incipiência neste quesito é algo exacerbado, apesar da importância desta temática para transição dos cuidados para o domicílio.

Apesar de sua relevância, em acesso a dispositivos de busca on-line, constatou-se uma escassez de jogos validados para orientações em educação em saúde, principalmente, tendo o público-alvo brasileiros no processo de desospitalização.

No processo de edificação desta pesquisa, desenvolveu-se as seguintes categorias:

\section{a. Papel da enfermagem na utilização dos jogos e das brincadeiras (Play and Playthings $x$ Nursing)}

De acordo com Schleisman e Mahon (2018), determinados diagnósticos de doenças podem ser uma experiência um tanto difícil para alguns pacientes que podem vir a apresentar sintomas de ansiedade, estresse, isolamento social, agressividade, depressão, dentre outros.

A enfermagem, exerce diversas funções, mas uma em destaque é o cuidado direto com os pacientes que visa o cuidado com o ser humano em si, de modo que busque preservar e promover a sua integridade aliviando o seu sofrimento e buscando uma melhor qualidade de vida (Dias, Domingues, Tibes, Zem-Mascarenhas \& Fonseca, 2018).

Pacientes pediátricos tendem a apresentar uma maior dificuldade em lidar com uma doença na qual necessite de um longo período de internação, como nas patologias oncológicas por exemplo, dessa forma, a enfermagem pode incorporar à rotina diária hospitalar diversas formas de atenuar esse sofrimento com a utilização de jogos e brincadeiras médicos e normativos que também ampliam o conhecimento desses pacientes a cerca de determinado tema (Ayed, et al., 2018). Vale ressaltar ainda, que a utilização dos jogos e das brincadeiras não são limitados somente a pacientes pediátricos, os adolescentes e adultos também podem ser incluídos nesse processo pela busca de minimizar o estresse, sofrimento e a ansiedade causados pelo tratamento de algumas doenças (Fonseca, et al., 2018).

Dessa forma, o brincar pode ser caracterizado como um tratamento alternativo para pessoas de todas as idades, pois, se aplicado da forma adequada, ele pode auxiliar esses indivíduos a lidar com as dificuldades psicossociais ou ainda impedí-las de desenvolver alguns distúrbios que podem agravar suas condições clínicas (Souza, et al., 2017).

A enfermagem tem um importante papel no alívio do sofrimento dos pacientes, pois ela consegue identificar os sintomas e as principais dificuldades enfrentadas tanto pelo paciente como pela família e com isso, são capazes de intervir da melhor forma para aliviar as emoções destes indivíduos. Os jogos e as brincadeiras beneficiam pacientes adultos ou pediátricos sempre com o mesmo objetivo: proporcionar o alívio do estresse vivido diariamente principalmente no ambiente hospitalar (Radhakrishnan, et al., 2016).

Em relação à utilização de jogos e brincadeiras, o enfermeiro deve oferecer a cada paciente a possibilidade de brincar de acordo com a sua faixa etária, ou seja, as brincadeiras oferecidas para crianças são diferenciadas daquelas dadas a adultos facilitando assim o processo de experiência. Os recursos utilizados por esses profissionais podem ser através de desenhos e pinturas, musicoterapia, brinquedos terapêuticos e outros que possam auxiliar na minimização da experiência do adoecimento e da hospitalização (Money, et al., 2019). 
Acredita-se que a intervenção da enfermagem nas brincadeiras melhora a adoção de comportamentos saudáveis e na autonomia dos pacientes, permitindo compreender como é importante o seu papel na vida dessas pessoas. A partir do momento que a enfermagem identifica os problemas e intervém sobre eles, o brincar pode estimular o paciente a enfrentar sua real situação, melhora sua aptidão fisica, mental, sua cognição, melhora sua auto-estima e também o mantém mentalmente e socialmente ativo conforme referem Costa et al. (2019).

Embora, as atividades lúdicas com jogos, brinquedos e brincadeiras possibilitem uma melhor qualidade de vida para indivíduos de todas as faixas etárias, a utilização desses recursos traz desafios diários para os profissionais de enfermagem, pois além de preparo para realizar tais atividades, necessitam ainda de recursos materiais que auxiliem no atendimento das particularidades de cada paciente o que nem sempre está disponível (Bock, et al., 2019).

O enfermeiro faz parte da classe que mais convive com o paciente no ambiente hospitalar e por isso tem uma maior capacidade em atender as necessidades do indivíduo como um todo e em promover um maior conforto e bem-estar. Sendo assim, este profissional utiliza os jogos e as brincadeiras para atender as necessidades emocionais do paciente, estabelecer vínculos e auxiliar no processo de vivência de uma doença. (Okitika, et al., 2015).

Os profissionais de enfermagem promovem a elaboração de estratégias de educação em saúde a fim de orientar os pacientes a cerca do processo saúde-doença através de uma ação educativa que apresenta um caráter de empoderamento que visa melhorar a qualidade de vida destes indivíduos (Olympio \& Alvim, 2018).

\section{b. O jogo como tecnologia educacional}

Devemos considerar que a brincadeira é imprescindível durante a infância, pois ajuda no desenvolvimento motor, emocional, social e mental das crianças, sendo ainda uma importante ferramenta para a sua comunicação e expressão de sentimentos como alegria, tristeza, frustrações, medo e ansiedade.

Nos últimos tempos, muitas coisas mudaram e com o avanço da tecnologia e da ciência, a área da saúde também passou por diversas transformações para a melhoria da assistência ao paciente, sendo a utilização de jogos, brinquedos e brincadeiras uma delas (Ayed, et al., 2018).

As atividades lúdicas facilitam o aprendizado, melhoram o desempenho do paciente trazendo benefícios à sua saúde através da interação e da utilização de processos tecnológicos que hoje estão inseridos em diversas áreas inclusive da saúde (Fernandes, Angelo \& Martins, 2018).

Como tecnologia educacional, os jogos são caracterizados como umas das atividades que mais aperfeiçoam a criatividade dos indivíduos através da dinâmica de um contexto educativo e da construção do conhecimento a partir da experiência em sua própria realidade (Rosi, et al., 2016).

A tecnologia educacional em saúde é fundamental para amenizar os efeitos devastadores do processo de hospitalização, uma vez que a mudança na rotina, submissão a procedimentos invasivos leva ao sofrimento do paciente e sua família, e por isso, se faz necessária a criação de estratégias amenizem essa situação (Bundy, et al., 2015).

Ainda de acordo com os autores, o uso de tecnologia educacional em saúde funciona como um potencializador nas atividades lúdicas realizadas no ambiente hospitalar. Diversos recursos podem ser utilizados para estimular os pacientes como desenhos, pinturas, músicas, uso de equipamentos eletrônicos para recurso áudio - visual entre outros.

Desenvolver tarefas que estimulem o ser humano, requer uma grande habilidade dos profissionais que devem identificar as maiores necessidades de determinados grupos e assim ter o discernimento de criar atividades que estimulem o desenvolvimento biopsicossocial de cada um para que todos se sintam ativos nesse processo. Acredita-se que o saber científico desde que vinculado a simples elementos como o cuidar, pode modificar o contexto pesaroso da doença e facilitar o processo de confiança e de vínculo entre paciente e os profissionais de saúde (Fonseca, et al., 2015). 
A associação entre o lúdico e a tecnologia educacional significa estimular a aprendizagem através de jogos e de brincadeiras que podem ser aleatórias ou com temáticas criando um ambiente gratificante e que propicia o desenvolvimento integral do indivíduo. Dessa forma, os jogos educacionais podem ser referidos como jogos assertivos projetados para mero entretenimento que incentivam a assimilação de novos conceitos e estimulam a aprendizagem (Vukićević, Đorđević, Glumbić, Bogdanović \& Jovičić, 2019).

No contexto hospitalar, a brincadeira pode ser caracterizada como um conceito de intervenção que constrói estratégias para auxiliar no enfrentamento de doenças, no processo de hospitalização, na comunicação com os profissionais envolvidos e na resolução de conflitos, além de estimular a expressão de sentimentos e emoções resgatando o 'ser' interior (Costa, et al., 2019).

\section{c. A educação em saúde por meio dos jogos, brinquedos e brincadeiras}

As brincadeiras, jogos e os brinquedos são ferramentas importantes que não devem apenas ser utilizadas como forma de estímulo para aliviar a tensão e o estresse, mas também como forma de auxiliar em uma melhor compreensão da situação vivenciada pelos pacientes, ou seja, os jogos podem ser utilizados como uma maneira de estimular o conhecimento e até mesmo esclarecer dúvidas quanto a prevenção de doenças, tratamento, sintomas, procedimentos necessários entre outros (Dickinson \& Wills, 2019).

Segundo Schleisman e Mahon (2018), com crianças hospitalizadas é muito comum a utilização de brinquedos terapêuticos que são manipulados pela própria criança a fim de facilitar o processo de comunicação entre ela e os profissionais de saúde permitindo uma melhor compreensão dos procedimentos a serem realizados. Este é um tipo de recurso que alivia as tensões da criança em relação ao tratamento proposto e que aproxima os profissionais do mundo infantil.

Já com pacientes adultos ou idosos, jogos de tabuleiros são bastante indicados como forma de terapia para aliviar a tensão e o estresse hospitalar. Para estes indivíduos, os jogos têm como objetivo permitir uma melhor aderência aos requisitos do tratamento como o uso de medicação contínua, minimizar distúrbios psicológicos, melhorar a comunicação, esclarecer dúvidas, oferecer conforto e melhoria da qualidade de vida (Tse, Kwan \& Lee, 2016).

Os jogos em saúde, funcionam como uma ferramenta educativa que consiste em refletir na cultura, que permite aos participantes construir um contexto sócio - histórico com a troca de experiências entre eles, apresentando um papel significativo no processo de aprendizagem e na construção da independência. Os jogos, portanto, são potencialmente capazes de desenvolver a educação e a construção dos conhecimentos em saúde, mas de forma interativa, estimulante, divertida e inovadora (Fonseca, et al., 2015).

Atualmente, os jogos em saúde são descritos como uma proposta de coparticipação, que estimula mudanças, e criam um local de reflexão e partilha de ações sobre suas próprias emoções. Os jogos permitem ainda a criação de um espaço onde buscam fortalecer os laços familiares sendo uma ferramenta de apoio para a aprendizagem com a construção de ideias e ações com grande eficácia na educação em saúde como jogos de perguntas e respostas que permitem avaliar o nível de conhecimento dos participantes a cerca de determinado tema (Bundy, et al., 2015).

Acredita-se que os jogos sejam importantes pois motivam e tornam possível a construção do pensamento crítico e reflexivo do indivíduo (Brandão, et al, 2019).

\section{Conclusão}

O enfermeiro vem como um mediador no processo educacional, utilizando o jogo educativo como uma tecnologia facilitadora para troca de conhecimento, criando um espaço para utilização da escuta ativa e sensível favorecendo o fortalecimento de vínculo, além da estruturação de saberes para alcance de mudanças de atitudes e hábitos para recuperação, 
prevenção e promoção da saúde. Facilita ainda o processo de entendimento sobre a sua real situação, contribui para o aumento da adesão ao tratamento e ainda promove habilidades para auxiliar no enfrentamento da doença e na melhoria da sua qualidade de vida.

As atividades lúdicas no ambiente hospitalar têm o intuito de promover a saúde e o enfrentamento da doença em situação de hospitalização não se restringindo apenas a ordem curativa. Os jogos têm uma importância na contribuição para a construção de vínculos entre pacientes e profissionais de saúde, facilitando o processo educacional através de uma comunicação dialógica, com intuito de troca de saberes para facilitar mudanças de comportamentos, autonomia e melhora na qualidade de vida. Levando em consideração o envolvimento do familiar/cuidador.

Vale ressaltar que a interação lúdica, independente da modalidade ou da faixa etária em que se é aplicada proporciona ao paciente um processo terapêutico menos doloroso e que assegure o seu bem-estar e a sua qualidade de vida.

Sugere-se novas pesquisas na área, utilizando jogos como ferramenta facilitadora para processo educacional, sendo utilizando em diversos cenários, com variabilidade de públicos, bem como, em diferentes níveis de atenção à saúde.

\section{Referências}

Ayed, I., Ghazel, A., Jaume-I-Capó, A., Moya-Alcover, G., Varona, J. \& Martínez-Bueso, P. (2018). Feasibility of Kinect-Based Games for Balance Rehabilitation: a case study: A Case Study. Journal Of Healthcare Engineering. p. 1-8. https://www.ncbi.nlm.nih.gov/pmc/articles/PMC6079427/.

Bock, B. C., Dunsiger, S. I., Ciccolo, J. T., Serber, E. R., Wu, W., Tilkemeier, P., Walaska, K. A. \& Marcus, B. H. (2019). Exercise Videogames, Physical Activity, and Health: Wii Heart Fitness: A Randomized Clinical Trial. Am J Prev Med, Rhode Island. p. 501-511. https://www.ncbi.nlm.nih.gov/p mc/articles/PMC7100962/.

Brandão, I. A., Whitaker, M. C. O., Oliveira, M. M. C., Lessa, A. B. S. L., Lopes, T. F. S., Camargo, C. L., Silva, J. C. \& Moraes, J. A. S. (2019). Jogos eletrônicos na atenção à saúde de crianças e adolescentes: revisão integrativa: revisão integrativa. Acta Paulista de Enfermagem. p. 464-469. FapUNIFESP (SciELO). https://www.scielo.br/scielo.php?pid=S0103-21002019000400464\&script=sci_arttext.

Bundy, A. C., Wyver, S., Beetham, K. S., Ragen, J., Naughton, G., Tranter, P., Norman, R., Villeneuve, M., Spencer, G. \& Honey, A. (2015). The Sydney playground project- levelling the playing field: a cluster trial of a primary school-based intervention aiming to promote manageable risk-taking in children with disability: a cluster trial of a primary school-based intervention aiming to promote manageable risk-taking in children with disability. Bmc Public Health. p. 1-5. Springer Science and Business Media LLC. https://bmcpediatr.biomedcentral. com.ez39.periodicos.capes.gov.b r/articles/10.1186/s12887-015-03 47-2.

Costa, P., Ermini, T. \& Sigaud, C. H. S. (2019). Effects of an educational playful intervention on nasal hygiene behaviors of preschoolers: a quasiexperimental study: a quasi-experimental study. Health Promotion Perspectives. p. 50-54. Maad Rayan Publishing Company. http://web-a-ebscohost.ez39.peri odicos.capes.gov.br/ehost/pdfvie wer/pdfviewer?vid=1\&sid=9cd4 abad-fe97-499d-9692-0f4ad4b30 099\%40sessionmgr4007.

Dias, J. D., Domingues, A. N., Tibes, C. M., Zem-Mascarenhas, S. H. \& Fonseca, L. M. M. (2018). Serious games as an educational strategy to control childhood obesity: a systematic literature review: a systematic literature review. Revista Latino-americana de Enfermagem. p. 1-10. https:/www.scielo.br/scielo.php?script=sci_arttext\&pid=S0104-11 692018000100608\&lng=en\&nrm =iso\&tlng=en.

Dickinson, A. \& Wills, W. (2019). Addressing malnutrition and access to food for older people: the Food In Later Life Game. Journal of Community Nursing. p. 16-17. http://web-a-ebsc ohost.ez39.p eriodicos.capes.gov.br/ehost/pdfviewer/pdfviewer?vid=9\&sid=ecfeac60-9b94-49e6a380dda68a9a6b\%40sessionmgr4006.

Fernandes, C. S., Angelo, M. \& Martins, M. M. (2018). Dar Voz aos Cuidadores: um jogo para o cuidador familiar de um doente dependente. : um jogo para o cuidador familiar de um doente dependente. Revista da Escola de Enfermagem da Usp. p. 1-8. https://www.scielo.br/scielo.php? script=sci_arttext\&pid=S0080-62 342018000100427\&lng=pt\&tlng =pt.

Fonseca, A., Santo, F. H. E., Silva, R. M. C. R. A. \& Silvino, Z. R. (2015). Games to promote health education for children: integrative review, Revista de Enfermagem Ufpe On Line, Recife. p. 8444-8452. http://web-b-ebscohost.ez39.periodicos.capes.gov.br/ehost/pdfvie wer/pdfviewer?vid=1\&sid=5704 a21c01f5-4465-9d86-b670f73bc 879\%40pdc-v-sessmgr05.

Fonseca, R. M. G. S., Santos, D. L. A., Gessner, R., Fornari, L. F., Oliveira, R. N. G. \& Schoenmaker, M. C. (2018). Gender, sexuality and violence: perception of mobilized adolescents in an online game: perception of mobilized adolescents in an online game. Revista Brasileira de Enfermagem. p. 607-614. https://www.scielo.br/scielo.php? script=sci_arttext\&pid=S0034-71 672018000700607\&lng=en\&nrm =iso\&tlng=en:

Mendes, K. D. S., Silveira, R.C.C.P. \& Galvão C.M. (2008). REVISÃO INTEGRATIVA: Método de pesquisa para a incorporação de evidências na saúde e na enfermagem. Texto Contexto Enferm. p. 758-64. https://www.scielo.br/j/tce/a/XzFkq6tjWs4wHNqNjKJLkXQ/?lang=pt\&format=pdf

Money, A. G., Atwal, A., Boyce, E., Gaber, S., Windeatt, S. \& Alexandrou, K. (2019). Falls Sensei: a serious 3D exploration game to enable the detection of extrinsic home fall hazards for older adults. Bmc Med Inform Decis Mak, Londres. p. 1-16. https://www.ncbi.nlm.nih.gov /pubmed/30992072.

Olympio, P. C. A. P. \& Alvim, N. A. T. (2018). Board games: gerotechnology in nursing care practice: gerotechnology in nursing care practice. Revista Brasileira de Enfermagem. p. 818-826. https://www.scielo.br/scielo.p hp?script=sci_arttext\&pid=S003 4-71672018000800818\&lng=en \&nrm=iso\&tlng=en. 
Okitika, T. A., Barnabé, R. V., Rue, T., Weisman, J., Harris, N. A., Orenstein, W. A. \& Wasserheit, J. N. (2015). "Polio Eradication" Game May Increase Public Interest in Global Health. Jornal Games for Health, Seattle. p. 195-201. https://www.ncbi.nlm.nih.gov/pu bmed/26182064.

Pereira, A. S., Shitsuka, D. M., Parreira, F. J., \& Shitsuka, R. (2018). Metodologia da pesquisa cientifica. [free e-book]. Santa Maria/RS. Ed. UAB/NTE/UFSM. https://www.ufsm.br/app/uploads/sites/358/2019/02/Metodologia-da-Pesquisa-Cientifica_final.pdf.

Radhakrishnan, K., Toprac, P., O'hair, M., Viés, R., Kim, M. T., Bradley, P. \& Mackert, M. (2016). Interactive Digital e-Health Game for Heart Failure SelfManagement: A Feasibility Study. Jornal Games for Health, Texas. p. 366-374. https://www.ncbi.nlm.nih.gov/p mc/articles/PMC5180075.

Rosi, A., Dall'asta, M., Brighenti, F., Rio, D., Volta, E., Baroni, I., Nalin, M., Zelati, M. C., Sanna, A. \& Scazzina, F. (2016). The use of new technologies for nutritional education in primary schools: a pilot study: a pilot study. Public Health. p. 50-55. https://www-sciencedirect.ez39.p eriodicos.capes.gov.br/science/article/pii/S0033350616302220?vi a\%3Dihub.

Schleisman, A. \& Mahon, E. (2018). Creative Play: a nursing intervention for children and adults with cancer. Clinical Journal of Oncology Nursing. p. 137140. Oncology Nursing Society (ONS). http://web-a-ebscohost.ez 39.periodicos.capes.gov.br/ehost/ pdfviewer/pdfviewer?vid=1\&sid =224b2dac-0695-4ae8b566-a76 6c21 fe $4 \mathrm{~d} 5 \% 40$ sessionmgr4006

Souza, V., Gazzinelli, M. F., Soares, A. N., Fernandes, M. M., Oliveira, R. N. G. \& Fonseca, R. M. G. S. (2017). The game as strategy for approach to sexuality with adolescents: theoretical-methodological reflections: theoretical-methodological re-ections. Revista Brasileira de Enfermagem. p. 376-383. https://www.scielo.br/scielo.php? script=sci_arttext\&pid=S0034-71672017000200376\&lng=en\&nrm =iso\&tlng=en.

Tossin, B. R., Souto, V. T., Terra, M. G., Siqueira, D. F., Mello, A. L. \& Silva, A. A. (2016). Educational practices and self-care: evidence in scientific production of nursing. Reme: Revista Mineira de Enfermagem. p. 1-8. https://pesquisa.bvsalud.org/portal/resource/pt/bde-28790.

Tse, I. M. Y., Kwan, T. S. \& Lee, P. H. (2016). The development and psychometric evaluation of the Perceptionof Play Questionnaire for older adults. Educational Gerontology, Hong Kong. p. 79-88. http://web-a-ebscohost.ez39.peri odicos.capes.gov.br/ehost/detail/ detail?vid=0\&sid=553a4935-232 b-49f3b3f5-8d61db7743cb\%40s essionmgr4006\&bdata=Jmxhbm c9cHQtYnImc210ZT1laG9zdC1s aXZl\#AN=112212976\&db=c8h.

Välimäki, M., Mishina, K., Kaakinen, K J., Holm, K. S., Vahlo, J., Kirjonen, M., Pekurinen, V., Tenovuo, O., Korkeila, J., Hämäläinen, H., Sarajuuri, J., Rantanen, P., Orenius, T. \& Koponen, A. (2018). Digital Gaming for Improving the Functioning of People with Traumatic Brain Injury: Randomized Clinical Feasibility Study. J Med Internet Res. p. 1 - 18. https://www.jmir.org/2018/3/e77/.

Vukićević, S., Đorđević, M., Glumbić, N., Bogdanović, Z. \& Jovičić, M. Đ. (2019). A Demonstration Project for the Utility of Kinect-Based Educational Games to Benefit Motor Skills of Children with ASD. Perceptual And Motor Skills. p. 1117-1144. http://web-b-ebscohost.ez39.peri odicos.capes.gov.br/ehost/pdfvie wer/pdfviewer?vid=1\&sid=8e4af 577-f120-4f1d-8c00-0c26040e09 44\%40pdc-v-sessmgr05.

Wu, J., Loprinzi, P. D. \& Ren, Z. (2019). The Rehabilitative Effects of Virtual Reality Games on Balance Performance among Children with Cerebral Palsy: A Meta-Analysis of Randomized Controlled Trials. Int J Environ Res Saúde Pública. p. 1-13. https://www.ncbi.nlm.nih.gov/p mc/articles/PMC6861947/. 